\title{
Strain Sensor-Embedded Soft Pneumatic Actuators for Extension and Bending Feedback
}

\author{
Michelle C. Yuen ${ }^{1,2,3}$, Rebecca Kramer-Bottiglio ${ }^{2}$, and Jamie Paik ${ }^{1}$
}

\begin{abstract}
For soft robots to leave the lab and enter unstructured environments, proprioception is required to understand how interactions in the field affect the soft structure. In this work, we present sensor-embedded soft pneumatic actuators (sSPA) that can observe both extension and bending. The sensors are strain sensitive capacitors, which are bonded to the interior of fiber-reinforced extension actuators on opposing faces. This construction allows extension and bending to be measured by calculating the mean and difference in sensor responses, respectively. The sSPAs are bonded together to form a flat fascicle to increase the force output and prevent buckling under load, and are robust to component failure by incorporating redundancy. In this paper, we discuss the fabrication of the sensors and their subsequent integration into the actuators. We also report the work capacity and sensor response of the SSPA fascicles under extension, bending, and the combination of both modes of deformation. The sensorembedded soft pneumatic actuators presented here will advance the field of soft robotics by enabling closed-loop control of soft robots.
\end{abstract}

\section{INTRODUCTION}

There is a continuing need in the field of soft robotics for sensing methods for soft actuators. These devices are typically fabricated from soft materials, in particular silicone elastomers which are flexible (Young's modulus $<10 \mathrm{MPa}$ in the linear regime) and stretchable (elongation at yield of $500 \%$ ) [1]. While having many beneficial properties, silicone elastomers are difficult to model because they exhibit nonlinear and time-dependent stress-strain behaviors, undergo continuum deformations, and have effectively infinite degrees of freedom [1], [2]. These characteristics make model-based or open-loop control challenging to implement and thus, state feedback information is often necessary to perform positional control of soft actuators. The field of soft sensing addresses this need by developing sensors for measuring and withstanding large deformations, with minimal impact on the behavior of the system. Integration of the sensor and actuator into the same volume can yield a better measurement of the actuator state as compared to designs wherein the sensor can experience motion relative to the actuator, and thus inaccurately report the actuator state.

Researchers have worked towards directly integrating sensors into pneumatic actuators for proprioception. A common approach is to embed highly stretchable strain gauges composed of liquid metal microchannels into the body of a

\footnotetext{
${ }^{1}$ Reconfigurable Robotics Laboratory, Institute of Mechanical Engineering, École Polytechnique Fédérale de Lausanne, Lausanne, Switzerland. ${ }^{2}$ School of Engineering and Applied Science, Yale University, New Haven, CT, USA. ${ }^{3}$ School of Mechanical Engineering, Purdue University, West Lafayette, IN, USA email: jamie.paik@epfl.ch
}

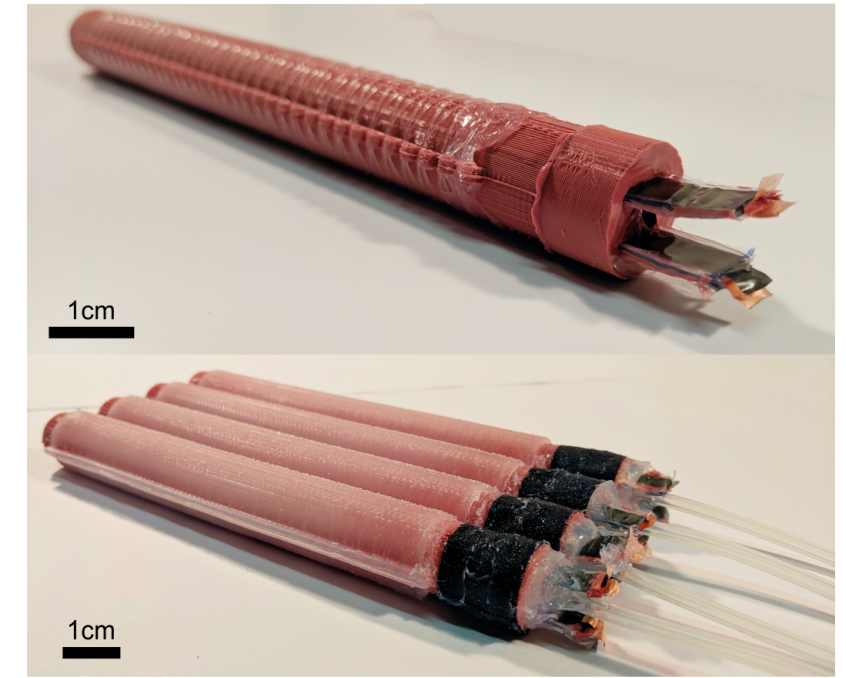

Fig. 1. Sensor-embedded soft pneumatic actuators (sSPA). (Top) Two capacitive strain sensors, with the interfaces protruding, are embedded along the interior cavity of a soft pneumatic actuator (SPA). (Bottom) A soft pneumatic actuator fascicle consisting of four parallel sSPAs to amplify force and reduce buckling.

silicone-based pneumatic actuator to achieve varying levels of feedback and control. Liquid metal microchannel sensors have been embedded in linearly contracting pneumatic artificial muscles as radial and axial sensors [3]-[6]. Bending actuators, including those used for soft pneumatic grippers, have been fabricated with liquid metal microchannels to create resistive sensors for determining bend angle, degree of grasp, or contact with an object [7]-[11]. By adding a redundancy of strain sensors around a pneumatic actuator (more sensors than degrees of freedom) and using machine learning techniques, researchers have shown that bending motions in 3D space can be reconstructed [11]. Other emerging approaches for sensing motion of pneumatic actuators are induction sensing with coiled wires to detect contraction of bellows actuators [12], [13], resistive sensing using silversilicone conductive composites to measure bending angle [14], magnetic sensing between joints using a magnetic silicone composite [15], optical waveguides for sensing strain along a pneumatic actuator [16], [17], and siliconebased capacitive sensors embedded within a fabric sleeve to measure the bend angle of a bellows-actuated robotic arm [18]. A model for using dielectric elastomer sensors for contraction sensing within a McKibben muscle described the deformation expected within the muscle and related it to the response of the sensor [19]. Finally, researchers have also 
utilized commercial, off-the-shelf components for curvature feedback on bending actuators. Some examples include the use of air pressure sensors connected to a deformable air void along the contacting surface of a pneumatic gripper [20], commercial optical fiber waveguides attached along the neutral axis of a bending actuator to measure curvature via light intensity [21], and resistive flex sensors attached on the inextensible layer of a bending actuator [22], [23]. All of these applications of commercially available devices are integrated in the non-extending region of the actuator, which is indicative of the lack of highly deformable sensors within commercially available devices.

The majority of the works cited here utilize an embedded sensor to measure a singular value of strain or curvature. While this approach is sufficient for pneumatic actuators operating in free space or within an expected protocol (such as grasping an object) in unstructured environments, the information provided by the sensors may not reflect the true configuration of the actuator. For example, consider a single actuator that encounters an unexpected obstacle during its programmed motion. A soft actuator can deform around the obstacle, and the deformation transduced by the sensor is a coupling between the undesired deformation and the commanded actuation. Therefore, there is a need to measure the deformation of pneumatic actuators such that their deformation can be measured even when operating outside of expected conditions.

In this work, we directly integrate highly deformable, capacitive strain sensors into soft pneumatic actuators (SPA) to create a sensor-embedded soft pneumatic actuator (sSPA) (Figure 1). By embedding a pair of displacement sensors into the SPA, we are able to determine distinctly both extension and bending by calculating the mean and difference of the two sensor measurements, respectively. This approach is similar to that reported by [24], wherein the sum and difference of the values reported from two curvature/strain sensors mounted back-to-back were used to report linear strain and curvature. Using the sSPAs presented here, the extension and bending angle can be determined from two sensor values and the pneumatic pressure applied to the actuators. By measuring both extension and bending, a better representation of the actuator deformation can be achieved, an important step towards feedback control of soft pneumatic systems in unstructured environments.

\section{FABRICATION}

\section{A. Sensors}

The sensors used in the sSPAs are closely based on previous work by White, et al. [25] which presented capacitive strain sensors fabricated in large multi-layer films from silicone-based conductive composite and silicone elastomer materials. In this work, we expanded upon the three-layer devices (conductive electrode - dielectric layer - conductive electrode) presented in [25] to a five-layer device (Figure 2(a,b)). This modification served two purposes: 1) it increased the size of the strain-sensitive capacitance by a
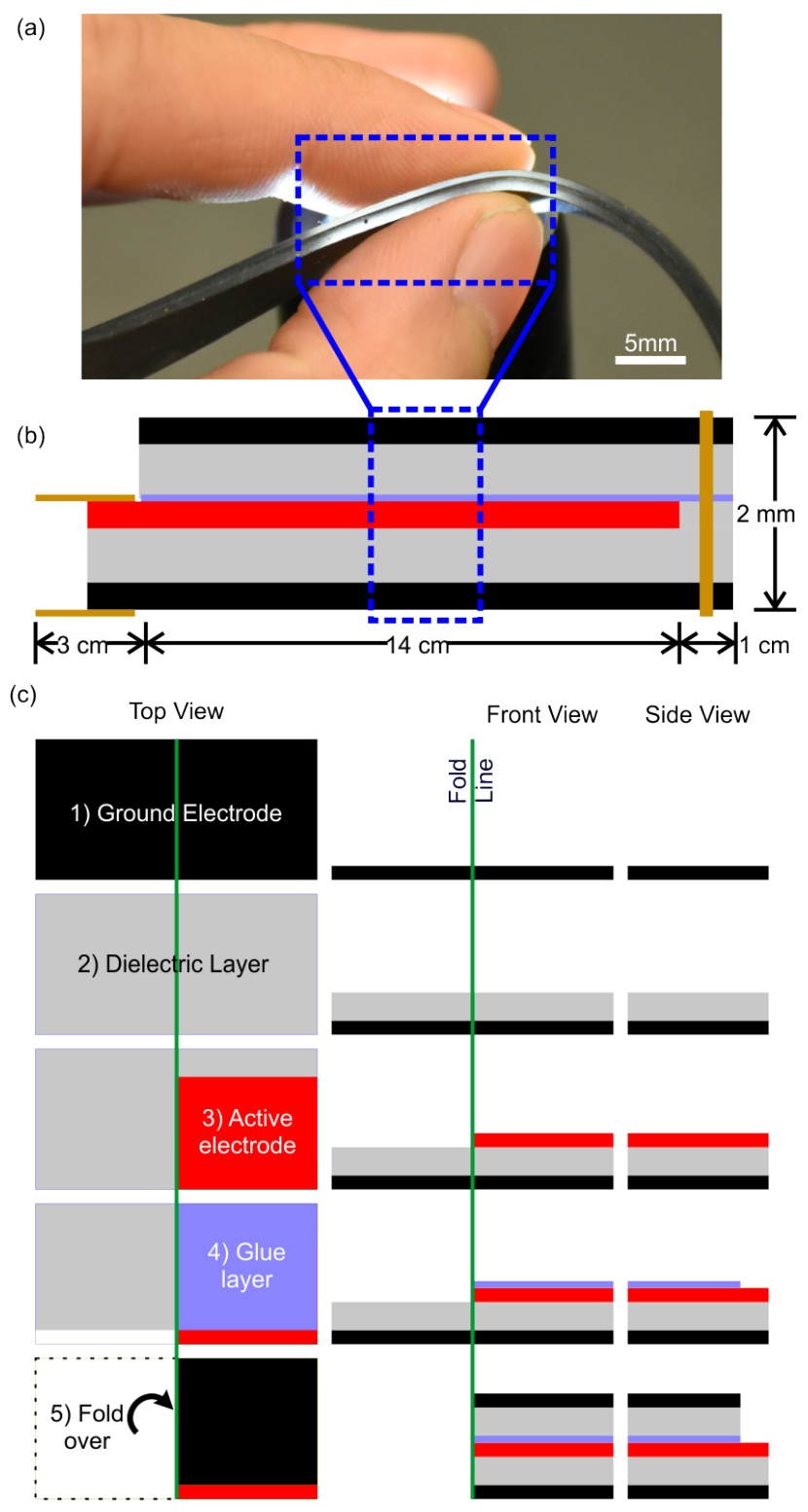

Fig. 2. Image and schematic of the sensor and fabrication process. (a) Photo of the cross-section of the sensor. (b) Schematic of the cross-section (side view) of a completed sensor. The layer types are indicated below in (c); the copper-colored regions indicate copper strips for interfacing the active and ground electrodes to the signal conditioning electronics, and copper wire to electrically connect the two ground electrodes together. (c) Top, front, and side views of the sensor film at various stages in the fabrication process.

factor of four, and 2) it shielded the charged layer from external electromagnetic noise.

Fabrication of the sensors requires five distinct processes: 1) creation of the conductive composite material for the electrode layers, 2) rod-coating the ground electrodes, dielectric layers, and active electrode layers, 3) folding the film onto itself to create a 5-layer capacitor, 4) cutting out sensors, and 5) interfacing with signal conditioning electronics. The conductive electrode layers were made from a composite of silicone elastomer and expanded graphite. The graphite was first expanded by placing $3 \mathrm{~g}$ of expandable graphite (Sigma Aldrich) into a ceramic crucible and then 

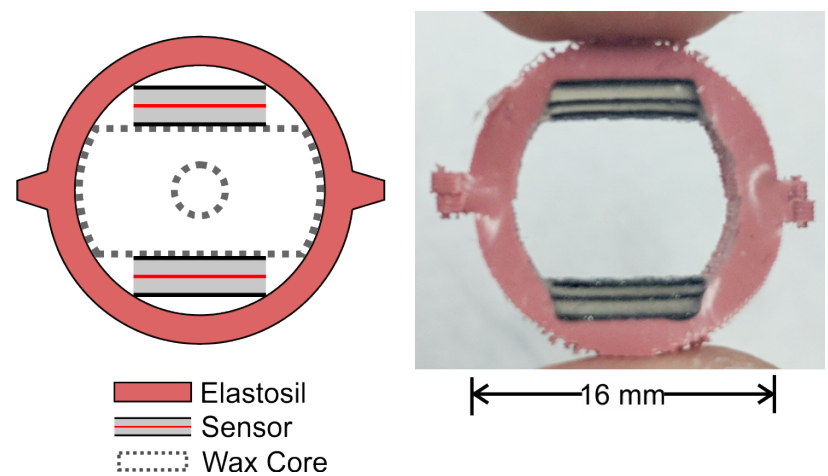

Fig. 3. Cross-sectional views of the sensor-embedded soft pneumatic actuator (SPA). The cross-section shows the two sensors bonded to the top and bottom interior of the SPA (shown in pink). The tabs on the side are used to secure the inextensible fishing line reinforcements. The sacrificial wax core is indicated by the dotted line; the center circle indicates the alignment rod used to center the wax core within the mold for the silicone.

roasting in a $450^{\circ} \mathrm{C}$ oven (Nabertherm B130) for 10 minutes. The expanded graphite was then mixed with $150 \mathrm{~mL}$ of cyclohexane in a glass bottle and then sonicated for a total energy deposition of $\approx 130 \mathrm{~kJ}$ (Vibracell Sonics VCX130, $100 \%$ amplitude, 1 hour). The mixture was filtered through a $220 \mu \mathrm{m}$ stainless steel sieve (Fisher Scientific) into a glass beaker and then dried until the concentration of expanded graphite in the cyclohexane mixture reached $\approx 6 \mathrm{wt} \%$. The graphite-cyclohexane slurry was mixed into uncured silicone elastomer (DragonSkin 10 Slow, Smooth-On Inc.) to obtain a final composite ratio of $10 \mathrm{wt} \%$ of graphite for $90 \mathrm{wt} \%$ silicon, after the cyclohexane evaporated.

Following preparation of the conductive composite material, the ground electrode, dielectric layer, and active electrode were rod-coated with a 1/2" Acme threaded rod onto a polyethylene terephthalate (PET) substrate (Figure 2(c)(1$3)$ ). Each film was rod-coated onto the previous film once it had fully cured. The active electrode was rod-coated onto half of the larger film, omitting a thin strip to allow the ground electrodes to be connected by a copper wire through the sensor (Figure 2(b) and (c)(3)). Following this step, the glue layer composed of native silicone elastomer was applied to the non-interfacing regions of the active electrode (Figure 2(c)(4)). While the glue layer was still uncured, the film was folded over and bonded onto itself to form a 5-layer structure (glue layer is omitted) (Figure 2(c)(5)).

Following completion of the sensor film, individual sensors were cut out of the film using a laser cutter (Laserscript, HPC Laserco.uk). The sensors were patterned such that the active region of the sensor (i.e., the full five layer portions) matched the active length of the actuators, thus leaving sufficient length for electrical interfacing to the sensor outside the actuator. The sensors were cleaned using soap and water. Copper wire was sewn through the end of the sensor to electrically join the two ground layers. Copper-clad polyimide film (Pyralux) and a PET backing was bonded to the active and one of the ground electrodes using a silicone adhesive (SilPoxy, Smooth-on). The sensors were then embedded in the actuators.

\section{B. Actuators with Embedded Sensors}

The design and manufacture of the actuators are closely based on previous work by Robertson, et al. [26]. The only modification to the fabrication described in [26] was to that of the sacrificial wax core. In this work, flats were added along the length of the wax core to accommodate the two sensors (Figure 3). After casting the wax core onto an alignment rod, the sensors were secured on the flats using a thin layer of silicone elastomer (EcoFlex 00-30, Smooth-On). Following this step, the wax core with sensors was inserted into the mold for the outer bladder of ElastoSil (M4601, Wacker Chemicals). After filling and degassing the mold, the actuators were allowed to cure for 12 hours. Because all the silicones used were platinum-cure silicone, the ElastoSil material fully-bonded to the sensors, ensuring that the sensor bodies did not move within the internal chamber of the actuator.

The sSPAs were then wrapped with inextensible fishing line (Trilene Monofilament $0.3571 \mathrm{~mm}$, Berkley) at a $57^{\circ}$ bias to cause extension when pressurized [26]. The mold contained tabs along the side of the actuator to space the fishing line and to hold it in place during extension and contraction. After wrapping the fiber reinforcement, the wax core was melted out of the core and a pneumatic line was inserted into one end. Silicone adhesive was used to seal the pneumatic line and to plug and the other end of the actuator. Four sSPAs were then bonded in parallel with EcoFlex 0030 to form a sSPA fascicle. In this way, the actuators have a multiplied force output and reduced tendency to buckle. Finally, signal conditioning boards were soldered onto each of the sensors to transduce the capacitance of each sensor into an analog voltage [25].

We chose to embed the sensors within the SPA in order to further isolate the sensors from electro-magnetic interference due to contact with charged bodies, such as human touch. We had conducted preliminary tests with the sensors bonded to the exterior of the actuators, but this resulted in large fluctuations in the sensor signal $(>100 \%$ of the full scale sensor response at maximum extension) upon touching the sensor. Furthermore, the more delicate conductive electrode layers are not subjected to abrasion by the fishing line during actuation and contraction cycles.

\section{CharacterizATION}

We performed a series of tests on the sSPA fascicle to characterize the actuation and sensing capabilities, namely the relationship between force, displacement, air pressure, and sensor response.

\section{A. Blocked Force Tests}

The blocked force tests simultaneously characterized the actuators' ability to do mechanical work at various air supply pressures and the sensors' responses to displacements and air pressures. The sSPAs were inflated at 50,100 , and $150 \mathrm{kPa}$ and the displacement was stepped in $5 \mathrm{~mm}$ increments using 
(a)

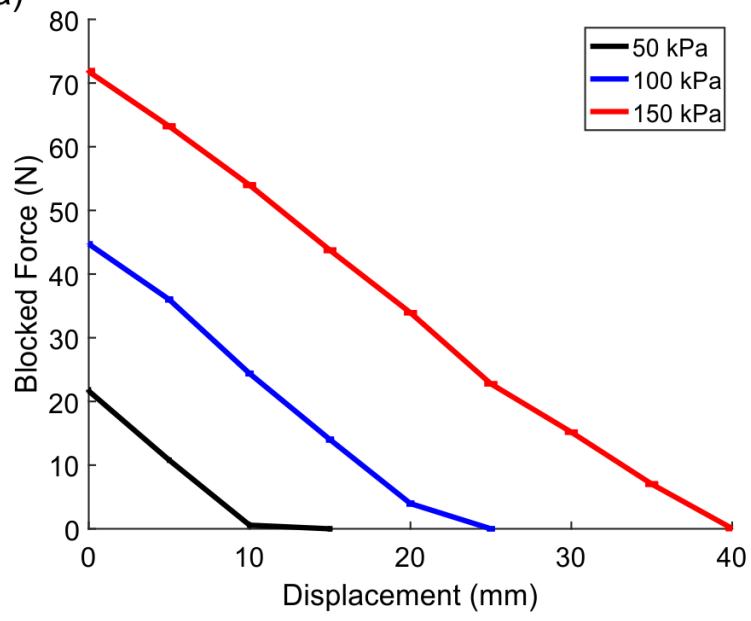

(b)

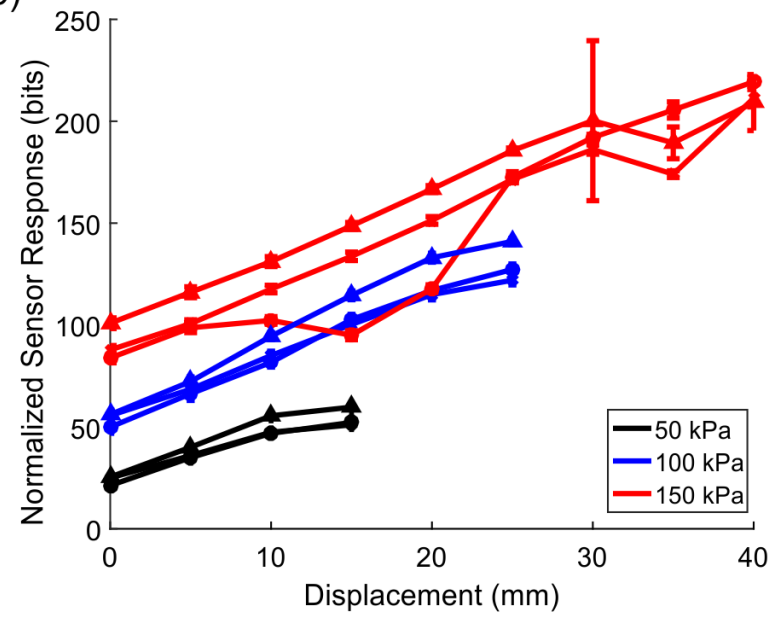

Fig. 4. Blocked-force characterization under linear extension of the sSPA fascicle. Each error bar represents the mean and 95\% confidence bounds of the value measured at steady state. (a) Force-displacement curves at pressures of 50, 100, and 150kPa. (b) Sensor responses corresponding to the same data points plotted in (a). The sensor values were normalized by subtracting the value measured when the actuator was unpressurized.

a linear stage, while the force was measured with a load cell and the sensor measurements were recorded via an Arduino Uno at a rate of approximately $10 \mathrm{~Hz}$. The sampling rate here was limited by the $8.4 \mathrm{~ms}$ charge-discharge cycle for measuring the sensors' capacitances. Each test was run at a specified pressure and fixed displacement. Upon beginning the test, the baseline, unpressurized state was recorded for 10s. The valve was then opened and the pressurized state was held for 20 s to allow the system to stabilize. Finally, the air was vented through the valve and the unpressurized state was recorded for a further 20 s.

The plots shown in Figure 4 show the force-displacement behavior and sensor response to displacement of the sSPAs at various pressures. Each error bar on the plot shows the mean and the $95 \%$ confidence bounds over 20 samples after the system had stabilized. The sensor responses were normalized by subtracting the initial value recorded while the actuator was unpressurized and unextended. As reported in [26], as the displacement increases, the delivered force reduces. At each pressure, we found that the force-displacement relationship is linear, indicating a constant work output at each inflation pressure. The sensor response plot shows that as the air pressure increases, the sensors' output signal increases, indicating an increase in capacitance due to compression. We also observed that the sensor response is sensitive to both the internal air pressure as well as the displacement, as demonstrated in the distinct offset of the curves corresponding to each pressure value in Figure 4(b). The sensors are composed of DragonSkin 10, a softer elastomer than the ElastoSil used in the bladder material, which will deform (i.e., compress in thickness) before the ElastoSil deforms (i.e., stretch). At a constant pressure, the sensor response-displacement response is approximately linear, as reported in [25]. Therefore, the embedded displacement sensors should ideally be used in conjunction with an air pressure sensor to decouple the effects of internal pressure and displacement. We hypothesize here that deviations away from the ideal linear fit, particularly at higher pressures, arise from instabilities in the interface between the sensor and the copper strips connecting to the signal conditioning electronics. Over the courses of these characterization tests, the actuators had a tendency to bend out of plane, particularly in tests with higher blocked forces. In some cases, these deformations resulted in the sensor partially losing contact with the copper strips, and in other cases, regaining contact with the copper strips. Improving the stability of the interface between the sensor and the signal conditioning electronics will be further studied in future work.

\section{B. Free Extension}

We also characterized the free extension length (Figure 5). These values correspond to the displacement at which zero force is exerted. Because the blocked force tests were performed in $5 \mathrm{~mm}$ increments, the true free displacement was not captured in Figure 4(a). As the pressure is increased linearly, the free displacement increases linearly as well, as measured by a time-of-flight sensor (SparkFun VL1680) (Figure 5(a)). This extension is tracked by the embedded sensors, to a certain extent (Figure 5(b)). Similar to the results in the blocked force tests, the sensors depart from their expected behavior at higher pressures, as shown by the larger error bars corresponding to the highest displacement at $200 \mathrm{kPa}$ inflation pressure. It should be noted however, that although the sensor signal at high pressures/displacements is less reliable, the sensors return to an operative state following this deformation.

\section{Bending Tests}

By embedding two strain sensors on opposite walls of the actuator chamber, we were able to measure bending by calculating the difference in sensor outputs of two sensors in the same actuator. In this test, the interface end of the fascicle was fixed while the other end was left free. A wooden dowel 
(a)

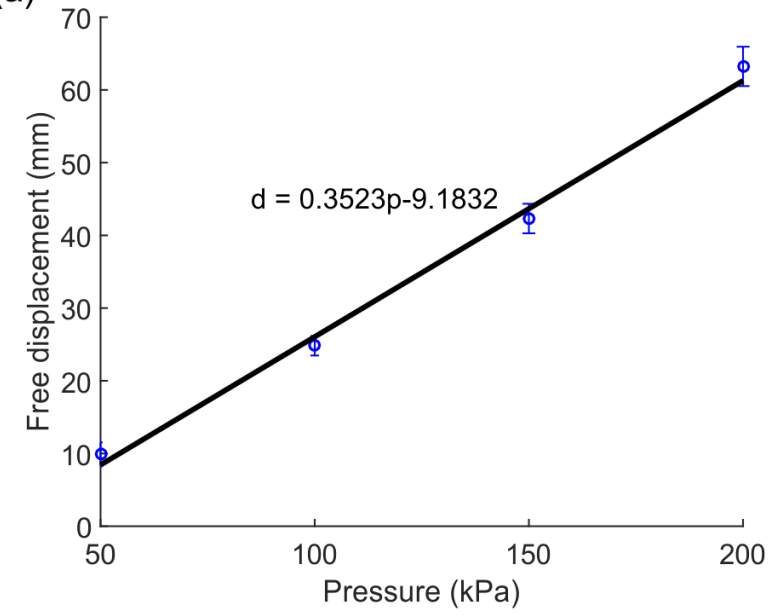

(b)

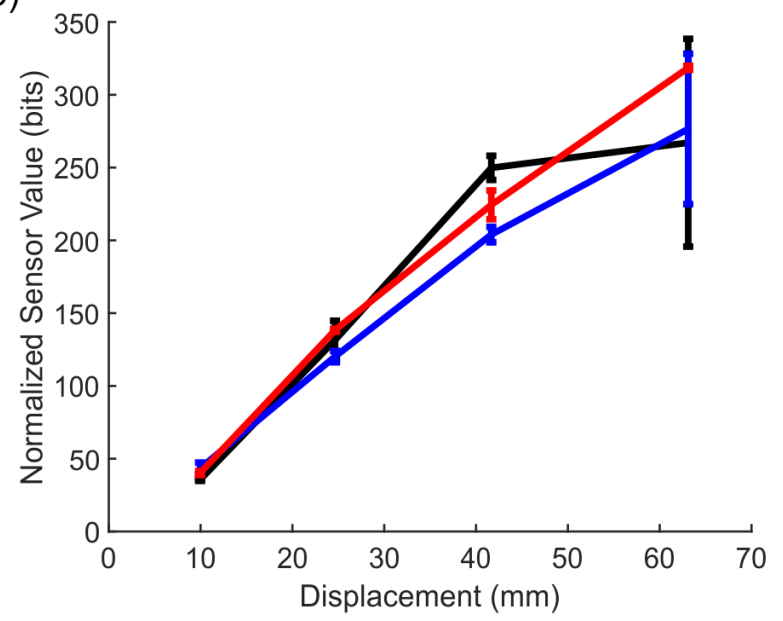

Fig. 5. Free displacement characterization under linear extension of the sSPA fascicle. Each error bar represents the mean and 95\% confidence bounds of the value measured at steady state. (a) Plot showing the free displacement lengths of the actuator as a function of pressure. The blue error bars are the measured data and the black line is the linear regression fit. (b) Responses of three sensors corresponding to the same data points plotted in (a). The sensor values were normalized by subtracting the value measured when the actuator was unpressurized. Note that the colors used to plot the data here do not correspond to those used in Figure 6.

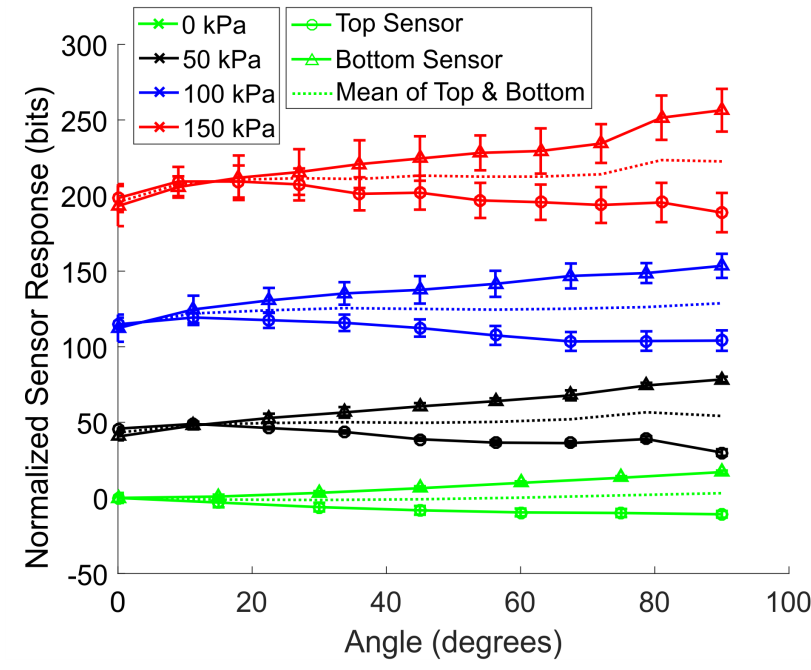

Fig. 6. Plot of the normalized responses of a sensor on the top and a sensor on the bottom of an SPA for different pressures. As the fascicle is bent upwards from a flat position, the top sensor contracts, while the bottom sensor stretches, resulting in the diverging sensor responses observed here. The mean sensor response stays relatively consistent throughout the bend, indicating that the overall length of the sensor is remaining the same throughout the bending.

was fixed crossing the fascicle at mid-length. The actuator was then inflated, and the bend angle was applied by raising the free end in $1 \mathrm{~cm}$ increments until a bend angle of $90^{\circ}$ was reached. The bend angle was measured as the angle between tangent lines drawn along each end of the fascicle. As the actuator is bent, the sensor on the outside of the bend (bottom of the SPA) is stretched, while the sensor on the inside of the bend (top of the SPA) is contracted, resulting in mirrored sensor responses, as seen in Figure 6. The sensor responses were normalized by subtracting the initial value recorded while the actuator was unpressurized and unextended. The sensor responses at a bend angle of $0^{\circ}$ correspond to the sensor responses at the final extension values shown in Figure 4, representative of the free extension length. As the bend angle increases, the two sensor responses diverge more and more, but the mean value of the two sensors stays consistent throughout the test. Therefore, by calculating the mean and difference in the sensor responses on either side of the sSPA and measuring the applied air pressure, the extension and bend angle may be distinctly determined.

\section{CONClusion AND Future Work}

In this paper, we have presented a soft pneumatic actuator with embedded strain sensors. By integrating two strain sensors along opposing faces in the interior chamber of an SPA, the extension and bending angle of the actuator can be ascertained by calculating the mean and differential responses of the sensors. These measurements enable a more accurate reconstruction of the state of the sSPA fascicle, which is particularly useful for applications of the sSPAs in environments where they will interact with its surroundings.

Future work will focus on further characterization of the SSPA's behavior, improving the mechanical robustness of the system, exploring different designs of sSPAs, and performing feedback control of the actuators using proprioceptive feedback from the sensors. In order to implement this in an integrated robotic system, it would be beneficial to more thoroughly investigate the dynamic behavior of the sSPAs. In particular, the bandwidth of the sensors and actuators, the effects of inflation rate, and performance under many cycles of operation will be relevant towards this goal. The mechanical robustness of the interface between the sensor body and the signal conditioning electronics was a limitation in this work. Because relative motion was allowed between the signal conditioning boards and the sSPAs, a great deal of stress was placed on the junction between the sensor and 
the copper strips. Some methods to potentially improve the interface are to reinforce the end of the sensor with fabric or to secure rigid plates around the interface. Additionally, the actuator robustness is sensitive to the tension applied to the fishing line as it is wound around the SPA. With uneven tension, the alignment of the weave around the actuator varied, allowing portions of the ElastoSil bladder to bubble up between the fishing line, which could lead to popping and failure of the actuator. Design of the sSPA can be tuned to fit the geometric requirements, motion trajectories, and force-displacement profiles needed by a given soft robotic system. The fabrication methods presented here can be easily scaled to accommodate various changes in geometry. An extension of the dual-sensor system presented here would be to incorporate three or more sensors to measure the full three degrees of freedom of the free end relative to the interface end (extension, bending about two axes). A variety of other silicone-based sensor types could also be incorporated on or in an SPA including torsional and exterior contact sensors. Lastly, control strategies may be applied to the current system presented here. Both position and force may be controlled using the results of the characterization tests presented here.

In conclusion, the work presented here on embedding a pair of sensors within a soft pneumatic actuator can advance the field of soft robotics by enabling better state reconstruction and control of a compliant actuator.

\section{ACKNOWLEDGMENTS}

The authors would like to thank Sagar D. Joshi for instruction on manufacturing the soft pneumatic actuators. This work was supported by the Swiss National Centre for Competence in Research (NCCR) Robotics and by the National Aeronautics and Space Administration (NASA) through a Small Business Technology Transfer Grant (Grant No. 80NSSC17C0030). MCY is supported by the National Science Foundation Graduate Research Fellowship (Grant No. DGE-1333468).

\section{REFERENCES}

[1] J. C. Case, E. L. White, and R. K. Kramer, "Soft Material Characterization for Robotic Applications," Soft Robotics, vol. 2, no. 2, pp. 80-87, June 2015.

[2] D. Rus and M. T. Tolley, "Design, fabrication and control of soft robots," Nature, vol. 521, no. 7553, pp. 467-475, May 2015.

[3] Y.-L. Park and R. J. Wood, "Smart pneumatic artificial muscle actuator with embedded microfluidic sensing," in SENSORS, 2013 IEEE. IEEE, 2013, pp. 1-4.

[4] Y. L. Park, B. r. Chen, C. Majidi, R. J. Wood, R. Nagpal, and E. Goldfield, "Active modular elastomer sleeve for soft wearable assistance robots," in 2012 IEEE/RSJ International Conference on Intelligent Robots and Systems, Oct. 2012, pp. 1595-1602.

[5] L. O. Tiziani, T. W. Cahoon, and F. L. Hammond, "Sensorized pneumatic muscle for force and stiffness control," in 2017 IEEE International Conference on Robotics and Automation (ICRA), May 2017, pp. 5545-5552.

[6] H. Kim, H. Park, J. Kim, K. J. Cho, and Y. L. Park, "Design of anisotropic pneumatic artificial muscles and their applications to soft wearable devices for text neck symptoms," in 2017 39th Annual International Conference of the IEEE Engineering in Medicine and Biology Society (EMBC), July 2017, pp. 4135-4138.

[7] R. A. Bilodeau, E. L. White, and R. K. Kramer, "Monolithic fabrication of sensors and actuators in a soft robotic gripper," in Intelligent Robots and Systems (IROS), 2015 IEEE/RSJ International Conference on. IEEE, 2015, pp. 2324-2329.
[8] N. Farrow and N. Correll, "A soft pneumatic actuator that can sense grasp and touch," in 2015 IEEE/RSJ International Conference on Intelligent Robots and Systems (IROS), Sept. 2015, pp. 2317-2323.

[9] Y. Hao, T. Wang, Z. Xie, W. Sun, Z. Liu, X. Fang, M. Yang, and L. Wen, "A eutectic-alloy-infused soft actuator with sensing, tunable degrees of freedom, and stiffness properties," J. Micromech. Microeng., 2017.

[10] J. Morrow, H. S. Shin, C. Phillips-Grafflin, S. H. Jang, J. Torrey, R. Larkins, S. Dang, Y. L. Park, and D. Berenson, "Improving Soft Pneumatic Actuator fingers through integration of soft sensors, position and force control, and rigid fingernails," in 2016 IEEE International Conference on Robotics and Automation (ICRA), May 2016, pp. 5024-5031.

[11] V. Wall, G. Zller, and O. Brock, "A method for sensorizing soft actuators and its application to the RBO hand 2," in 2017 IEEE International Conference on Robotics and Automation (ICRA), May 2017, pp. 4965-4970.

[12] W. Felt, K. Y. Chin, and C. D. Remy, "Contraction Sensing With Smart Braid McKibben Muscles," IEEE/ASME Transactions on Mechatronics, vol. 21, no. 3, pp. 1201-1209, June 2016.

[13] W. Felt, M. Telleria, T. Allen, G. Hein, J. Pompa, K. Albert, and C. D. Remy, "An Inductance-Based Sensing System for Bellows-Driven Continuum Joints in Soft Robots," in Proceedings of Robotics: Science and Systems, Cambridge, Massachusetts, 2017, oCLC: 853458128.

[14] J. C. Yeo, H. K. Yap, W. Xi, Z. Wang, C.-H. Yeow, and C. T. Lim, "Flexible and Stretchable Strain Sensing Actuator for Wearable Soft Robotic Applications," Adv. Mater. Technol., vol. 1, no. 3, pp. n/a-n/a, June 2016.

[15] M. Luo, Y. Pan, E. H. Skorina, W. Tao, F. Chen, S. Ozel, and C. D. Onal, "Slithering towards autonomy: a self-contained soft robotic snake platform with integrated curvature sensing," Bioinspir. Biomim., vol. 10, no. 5, p. 055001, 2015.

[16] S. Sareh, Y. Noh, M. Li, T. Ranzani, H. Liu, and K. Althoefer, "Macrobend optical sensing for pose measurement in soft robot arms," Smart Mater. Struct., vol. 24, no. 12, p. 125024, 2015.

[17] H. Zhao, K. OBrien, S. Li, and R. F. Shepherd, "Optoelectronically innervated soft prosthetic hand via stretchable optical waveguides," Science Robotics, vol. 1, no. 1, p. eaai7529, Dec. 2016.

[18] M. C. Yuen, H. Tonoyan, E. L. White, M. Telleria, and R. K. Kramer, "Fabric sensory sleeves for soft robot state estimation," in 2017 IEEE International Conference on Robotics and Automation (ICRA), May 2017, pp. 5511-5518.

[19] N. C. Goulbourne, S. Son, and J. W. Fox, "Self-sensing McKibben actuators using dielectric elastomer sensors," vol. 6524, 2007, pp. 652 414-652414-12.

[20] H. Yang, Y. Chen, Y. Sun, and L. Hao, "A novel pneumatic soft sensor for measuring contact force and curvature of a soft gripper," Sensors and Actuators A: Physical, vol. 266, no. Supplement C, pp. 318-327, Oct. 2017.

[21] H. Zhao, R. Huang, and R. F. Shepherd, "Curvature control of soft orthotics via low cost solid-state optics," in 2016 IEEE International Conference on Robotics and Automation (ICRA), May 2016, pp. 40084013.

[22] K. Elgeneidy, N. Lohse, and M. Jackson, "Bending angle prediction and control of soft pneumatic actuators with embedded flex sensors A data-driven approach," Mechatronics, Oct. 2017.

[23] G. Gerboni, A. Diodato, G. Ciuti, M. Cianchetti, and A. Menciassi, "Feedback control of soft robot actuators via commercial flex bend sensors," IEEE/ASME Transactions on Mechatronics, vol. PP, no. 99 pp. 1-1, 2017.

[24] E. L. White, J. C. Case, and R. K. Kramer, "Multi-mode strain and curvature sensors for soft robotic applications," Sensors and Actuators A: Physical, vol. 253, pp. 188-197, Jan. 2017.

[25] E. L. White, M. C. Yuen, J. C. Case, and R. K. Kramer, "Low-Cost, Facile, and Scalable Manufacturing of Capacitive Sensors for Soft Systems," Adv. Mater. Technol., vol. 2, no. 9, pp. n/a-n/a, Sept. 2017.

[26] M. A. Robertson, H. Sadeghi, J. M. Florez, and J. Paik, "Soft Pneumatic Actuator Fascicles for High Force and Reliability," Soft Robotics, Oct. 2016. 